\title{
7. Die Religion
}

Vor der Einführung des Christentums wurde der altorientalische Kult der Muttergöttin Kybele wie im übrigen Kleinasien auch in Isaurien praktiziert; Sallust erwähnt für das 1. Jahrhundert v. Chr. einen der Mater Magna geweihten Berg vor der Polis Isaura Nova.' Von ausgeprägtem religiösem Empfinden zeugen die prächtigen, oft im Kontrast zur Dürftigkeit der ländlichen Siedlungen stehenden isaurischen Grabinschriften und Sarkophage. ${ }^{2}$ Sie datieren kontinuierlich von der hellenistischen bis in die römische Zeit. Ihre Dekorationen sind eigenständig, nur die abgebildeten Löwen führen die anatolische Tradition fort. ${ }^{3}$ Die Behauptung des Verfassers der Vita Cononis, daß in diesem Landstrich die Idolatrie besonders verbreitet sei, ${ }^{4}$ ist wohl ein Widerhall der reichen isaurischen Sakralkunst gewesen.

Von einer frühen Christianisierung Isauriens bereits durch Paulus und Barnabas auf ihrer Reise nach Iconium berichten die Apostelgeschichte und die apokryphen Paulsakten mit der bereits oben vielfach erwähnten isaurischen Heiligen und ProtoMärtyrerin Thekla. Paulus reiste nach deren Zeugnis mit der Jungfrau Thekla in Isaurien und Lykaonien umher. Sie vollbrachte ihrerseits Wundertaten. Schließlich soll er sie mit der Verkündigung des Evangeliums in dieser Gegend beauftragt haben. Sie ist die erste Märtyrerin der Kirche. Sichere biographische Informationen über Thekla sind jedoch wegen der vielfältigen legendarischen Überwucherungen nicht zu gewinnen. ${ }^{5}$

Gerade an der Thekla-Figur läßt sich aufzeigen, daß es auch in Isaurien nicht zu einem radikalen Schnitt von den alten Religionen zum Christentum gekommen ist. ${ }^{6}$ Denn Thekla, wenngleich sie in der Hagiographie als Widersacherin und Bezwingerin der heidnischen Kulte gefeiert wird, stand in vielfältiger heidnischer Tradition. Offenbar flossen hier die Attribute mehrerer vorchristlicher weiblicher Gottheiten zusammen. Sie hat Funktionen der alten anatolischen Muttergottheit Kybele übernommen. Zahlreiche Darstellungen ihres zweiten Märtyriums, der Verurteilung ad bestias

1 Sall. hist. frg. II 87: [Servilius] occupavit sacrum Matri Magnae; WE 150; vgl. Friedrich SCHWENN, s.v. 'Kybele', RE XI 2 (1922) 2250-2298. 2267. 2286.

2 RAMSAY (1904) passim; DAGRON Mir. (1978) 120; BUSCHHAUSEN (1971)

3 RAMSAY (1904) 289-292; VERZONE (1959) 16 mit Abbildungen; MitFORD (1980) 1255 spekuliert über eine daraus resultierende Faszination am Tod; zur Dekoration der Grabbauten: ER (1991).



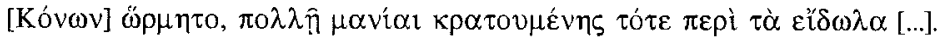

5 Apg. 13f.; Acta Pauli et Theclae 40f. = NT Apo II 20. 25. 216-224; TIB 5,1 85 f; zur Wirkungsgeschichte GianNARELLI (1991); zum Kult Davis (2001); allg. Klaus-Gunther Wesseling, s.v. 'Thekla', BBKL XI (1996), 806-814.

6 MiTFORD (1990) 2157f. zur geringen Aussagekraft der Inschriften und Grabbauten; die heidnischen Kultzentren „,were small, not endowed with wide estates and wealth.“ 
(aus dem sie abermals unversehrt hervorging) mit den Löwen auf Grabreliefs, Reliquiaren und Pilger-Ampullae, wo sie mit großen Brüsten abgebildet ist, lassen auf diesen alten heidnischen Aspekt schließen. ${ }^{7}$ Darüber hinaus besaß sie Züge der Athene, der vormaligen Schutzgöttin von Seleucia: Wenn die Stadt besser als andere den Anstürmen der isaurischen Banditen standhalten konnte, so war dies nach den im frühen 5. Jh. verfaßten Miracula vor allem dem Beistand der Heiligen zu danken. Die Miracula wurden zwischen 430 und 470 verfaßt. ${ }^{8}$ Der anonyme Autor des Werks ist wohl ein ehemaliger Rhetor, der dann Priester geworden war. ${ }^{9}$ Er verlängerte die bekannte Biographie der Thekla mit einer Erzählung ihrer zeitgenössischen Wundertaten in die Gegenwart, um die Wundertätigkeit der Thekla seinen Zuhörern in einem gegenwärtigen Zeitpunkt und einer limitierten geographischen Zone näherzubringen. ${ }^{10}$ Gregor von Nazianz verglich sie mit Athene und nannte das Heiligtum der Thekla ohne weiteres „Parthenon“." Auch der Artemis-Kult floß in die Heilige ein,

7 Zu den Löwenattributen und ihrer Verbindung mit Kybele vgl. Michael RiEMENSCHNEIDER, 'Heidnische Götter in christlichem Gewande. Die Löwenheiligen' in: Johannes IRMSCHER (Hrsg.), Byzantinische Beiträge, Berlin 1964, 81-90, gefolgt von BuschHAuSEN (1971) 196, dort die Reliquiar-Abbildungen. Zu Abbildungen von Heiligen und Märtyrern mit Tieren vgl. Henri LeCLERCQ, s.v. 'Ad bestias', DACL I 1 (1924) 449-462. 452 (Thekla). $\mathrm{Zu}$ den in der ägyptischen Menasstadt gefundendne ampullae vgl. DAvIS (2001); s.u. Kap. V.3.4.

8 Vg1. Dagron Mir. (1978) 115-123. Mir. 27 u. 28 erwähnen Attacken auf SelinuntTraianopolis (westlich von Anemurium an der Küste gelegen), vgl. Marc. com. s.a. 441; Dagron Mir. (1978) 119. Die in Mir. 13 erwähnte Exekution des Priesters Severus durch Saturninus, den Enkel des Saturninus unter Valens, im Jahr 444 in Jerusalem [die beiden waren Begleiter von Athenais-Eudokia und hatten sich häretischer Bemerkungen schuldig gemacht], vgl. Marc. com. s.a. 444; Cedr. I p. 601 f.; sowie die Bischöfe von Seleucia Dexianus, Johannes, Basilius und Porphyrius. Kaiser Zeno und seine Kirche in Hagia Thekla von 476 dagegen sind noch nicht erwähnt, vgl. DAGRON Mir. (1978) 16 ff., 323, Anm. 2.

9 DAgRon Mir. (1978) 15f., 140f.; zuerst dargelegt in: 'L'auteur des Actes et des Miracles de Sainte Thècle' in: $A B 92$ (1974), 5-11. Bischof Basilius ist nicht der Autor, wie es der griechische Titel in der Handschrift Vaticanus gr. 1853, einem Doppel-Palimpsest in Minuskelschrift aus dem frühen 10. Jh. (BHG 1717-1718), worin sie zusammen mit den apokryphen Paulsakten ( $B H G$ 1710) überliefert sind, nahelegt. Vielmehr handelt es sich bei dem Werk um eine Polemik gegen den Bischof von Seleucia, der ihn exkommuniziert hatte, Mir. 12, 41-110; und sein Nachfolger Porphyrius, Bischof wohl ab 468, hatte dies nicht aufgehoben, was dessen Verfluchung im Epilog nahelegt: Er stand selbst noch in der heidnischen Tradition, deshalb ist, so DAGRON Mir. (1978) $5 \mathrm{f}$., Vorsicht geboten mit einer Verallgemeinerung, welche Thekla schlicht als eine christliche Athena sieht. Die hier überlieferte $V$ ita ist eine Überarbeitung der von ihrem Autor als zu trocken erachteten $A C$ ta Apostolorum Apocrypha: DAGRON Mir. (1978) 22, 140-151; GOTTER (2003) passim.

10 DAgRon Mir. (1978) $20 \mathrm{ff}$.

11 Mir. 17; Greg. Naz. carm. I 547. 


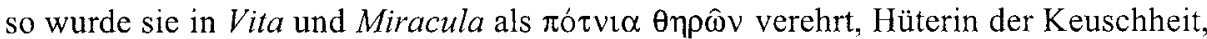
Beschützerin von Natur und Tieren. ${ }^{12}$

Auf der Teilnehmerliste des Konzils von Nicaea (325) sind erstmals isaurische Bischöfe bezeugt. Die Organisation und der Zusammenhalt der isaurischen Bischöfe setzten sich in der Spätantike fort. ${ }^{13}$ Die Isaurier insgesamt scheinen orthodox geblieben zu sein: Basilius von Caesarea beklagt in einem Schreiben an Eusebius von Samosata den „Verlust" von Tarsus - wohl an die heterodoxen Homöer. ${ }^{14}$

Die Religion gilt seit der Einführung des Christentums als alleiniger Reichsreligion durch Theodosius ${ }^{15}$ als eines der wichtigsten Integrationskriterien für die Kulturen im Reich. Nun gibt es eine Reihe von Quellen, die Heidentum bei den Isauriern bzw. Kontakte prominenter Isaurier zum Heidentum auch in der Spätantike bezeugen. In der Forschung werden diese Zeugnisse zuweilen als Anzeichen für deren fortgesetzten Widerstand gegen Rom und ihre Andersartigkeit gegenüber der Zivilisation des christianisierten Imperium Romanum interpretiert. ${ }^{16}$ Doch eindeutige Urteile über die Rolle der Religion bei dem Konflikt zwischen Isauriern und römischem Reich lassen

12 MAMA II 3f.

13 Zu den isaurischen Bischöfe in Nicaea vgl. Honigmann (1939) 48; TIB 4 86. 5,1 86. Beim Chalkedonense: $A C O$ II 1, 2 [348]; am Ende eines Schreibens des Bischofs Basilius von Seleucia an Kaiser Leo im Jahr 458: $A C O$ II 5 Nr. 27, p. 46-50. Liste: 49f.; Basil. ep. 190 zur Neubesetzung von isaurischen Bistümern Mitte des 5. Jhs.; sie sind ganz selbstverständlich in die Kirchenorganisation eingebunden: Basil. ep. 204, 7.

14 Basil. ep. 34 (ca. 369).

15 CTh XVI 1, 2.

16 Anzuzweifeln sind die für ein verbreitetes Heidentum bei den spätantiken Isauriern einstehenden Argumente von Trombley I (1993) 81 ff. (angebliche Gesetze des Kaisers Zeno gegen die Heiden in Verbindung mit der Rolle des Pamprepius beim Illus-Aufstand; zur Religionspolitik Zenos s.u. Kap. V.3.4.); Trombley II (1994) 120-129. 127 (Religiöse Verhältnisse in Kappadokien und Isaurien). Trombley geht von einer Christianisierung Isauriens nicht vor dem Jahr 400 aus und stellt die These auf, daß der unter Zeno errichtete Baukomplex Alahan hierfür gedient haben soll. Des weiteren bringt TromBLEY II (1994) 127 die bei Amm. XIV 2, 1 erwähnte Exekution der isaurischen Banditen in Iconium im Jahr 354, (,mass execution", wohl aus Hopwood (1986) 352) in Verbindung zu deren angenommenem Heidentum: ein Gesetz des Constantin (sein Verweis auf JoNES (1964) II 1017f. geht ins Leere) habe speziell verboten, daß Christen auf diese Weise hingerichtet werden. Doch bei Constantins Gesetz CTh IX 40, 2 handelt es sich lediglich um ein Verbot der zusätzlichen Brandmarkung von ad bestias Verurteilten; vgl. DEMANDT (1989) 76. Trombley bezieht sich wohl auf CTh IX 40, 8 des Valentinian und Valens, wonach kein verurteilter Christ in der Arena sterben dürfe. Dieses Gesetz jedoch ist erst 365, also elf Jahre nach den Ereignissen von Iconium erlassen worden und kann somit kaum etwas mit ihnen zu tun gehabt haben. Amm. l.c. will mit seiner Formulierung, die Hinrichtung sei praeter morem gewesen, weniger die Gesetzes- als die Sittenwidrigkeit dieser Bestrafung unter dem Regime des als tyrannisch gezeichneten Caesars Gallus andeuten; vgl. Demandt (1989) 84 u. Kap. IV.2.1.1. u. IV.3.1.2. zum Vorfall. 
sich bei genauerer Betrachtung nicht fällen. Dies beginnt schon bei der Schwierigkeit, den Grad der Christianisierung Isauriens in der Spätantike genauer zu ermitteln.

Sähe man die religiöse Differenz als ein Entscheidungkriterium für einen unüberbrückbaren Gegensatz zwischen Isauriern und Römern an und würde damit, wie zuletzt Brent SHAw, nach einer kulturellen oder ethnischen Ursache für den isaurischen Widerstand gegen Konstantinopel suchen, so ergäben sich zwei Argumentationsweisen, eine geographische und eine soziologische:

Als geographisches Argument für eine solche Unterscheidung hätten sich die alten Kulte vor allem im isaurischen Hinterland lange gehalten. ${ }^{17}$ In der Tat gibt es Hinweise dafür: Ein Flavius Indacus und sein Sohn Kottunes, mit Sicherheit identisch mit jenen Banditen, welche der spätere Kaiser Zeno während seines Heermeisteramtes zu bekämpfen hatte, machten eine Dedikation an einen Tempel nahe ihrer Festung Papirion. ${ }^{18}$ Einen Hinweis auf heidnisch gebliebene Isaurier gibt auch der erste Brief des Basilius an Aphilochius, den auf sein Betreiben eingesetzten Bischof von Iconium; er enthält die Aufforderung, „Menschen für den Herrn zu fangen und nach seinem Willen die vom Teufel Gefangenen aus der Tiefe heraus ans Licht zu ziehen," womit neben den Homöern wohl auch die heidnisch gebliebenen Isaurier gemeint sind. ${ }^{19}$

Aufgrund ihres Heidentums nun hätten die isaurischen Banditen die christlichen Stätten nicht respektiert. Die Basis für ein solches Argument wäre der Bericht der Pilgerin Egeria, worin die Befestigung des Thekla-Heiligtums erwähnt ist, ${ }^{20}$ und die 50 Jahre später verfaßten Miracula der Heiligen Thekla. In der Tat waren die Städte das Ziel der räuberischen Attacken. Doch für eine andere Religion der Isaurier insgesamt, von Einzelfällen wie den eben genannten bekennenden Heiden Indacus und Kottunes abgesehen, geben weder der Pilgerbericht der Egeria noch die Miracula (in welchen auch die Isaurier nie direkt benannt, sondern immer nur als "gefährliche Nachbarn“ oder „Plagegeister“ bezeichnet sind), ${ }^{21}$ noch die oben erwähnte Mönchsgeschichte des Theodoret einen Hinweis. Es war also wohl mehr der reiche Kirchenschatz des Heiligtums, der die Begehrlichkeiten der Isaurier weckte, als daß die kultische Funktion des Ortes sie zum Widerstand gereizt hätte.

Gegen eine religiöse Trennungslinie zwischen urbanisierter Ebene und angeblich nicht urbanisiertem Gebirge ${ }^{22}$ spricht eine weitere Nachricht: Die noch im 5. Jh. über-

17 So HellenKemper (1986) 630; LeNSKI (1999b) $323 \mathrm{ff}$.

18 BM II 146f. Nr. 148 schließen aus dem in der Inschrift bezeugten Heidentum auf eine Zeit vor Theodosius I., was keineswegs zwingend ist. Zu Indacus vgl. Suda I 368; Joh. Ant. frg. 206, 2. 214, 6. 10; PLRE II 590f. s.n. 'Indacus Cottunes'.

19 Basil.ep. 161.

20 Pereg. Aeth. XXIII 4.

21 Mir. 13.

22 Die vor allem seit Strabo eingeführte Opposition zwischen Ebene und Gebirge und deren 
arbeitete Vita der heiligen Thekla berichtet von einer heftigen Auseinandersetzung zwischen Christen und Anhängern des Sarpedonius-Kultes zur Zeit der Apostel. Thekla hatte gleichsam eine Bastion gegen den „Dämon“ Sarpedon-Apollon an der Küste und die gegen die Athena auf der Akropolis von Seleucia errichtet. Ihre erste Wundertat war, das Orakel des Apollo Sarpedonius zum Schweigen zu bringen. ${ }^{23}$ Der Tempel des Gottes befand sich bei der Bucht von Taşuçu beim Kap Sarpedon (İncekum Burun) südlich von Seleucia. Heute gibt es keine Überreste mehr; am Ort befindet sich die frühmittelalterliche Kirche des heiligen Theodoros. ${ }^{24}$ Das Orakel war nichtsdestoweniger noch im 3. Jahrhundert in öffentlicher, vielleicht sogar noch in der Spätantike in geheimer Funktion. ${ }^{25}$ Nach einer fragmentarisch erhaltenen koptischen Heiligenvita soll es dort sogar noch im 4. Jahrhundert zu heidnischen Kulthandlungen gekommen sein. Nach der Vita war der heilige Athanasius von Alexandria nach Seleucia gelangt, wo gerade ein Konflikt zwischen Christen und Heiden eskalierte. Während einer Feier für den Apollo Sarpedonius in einem Tempel sei es zu einem Felssturz gekommen, in dem alle darin befindlichen Heiden umgekommen seien. Daß es sich bei dieser Episode um einen hagiographischen Anachronismus handelt, mithin eine Verlegung von Zuständen aus der Zeit der Christianisierung in die Spätantike, kann bei dieser Quellengattung zwar nicht ausgeschlossen werden, doch macht die Erwähnung des Orakels bei Zosimus ein Fortleben des Apollo-Kultes gerade in der Provinzhauptstadt Seleucia glaubhaft. ${ }^{26}$

Einfluß auf den Charakter der Bevölkerung erweist sich bei näherer Betrachtung als nicht tragfähig; der Urbanisierungsgrad in den hochgelegenen Teilen Isauriens ist nicht geringer als in der Ebene, hierzu s.u. Kap. III.2.4, u. IV.3.2.2.

23 Vgl. Dagron Mir. (1978) 278, 290; MacKay (1990) 2110-2113, die Autorin hat leider die Arbeit von DAGRON nicht einsehen können und vermutet in den Miracula noch die Autorschaft des Basilius von Seleucia.

24 Ed. Oscar v. LemM, 'Koptische Fragmente zur Patriarchengeschichte Alexandriens' in: Mémoires de l'Académie Imperiale des Sciences de St.-Pétersbourg, VIIe Série, XXXVI, 11. St.-Pétersbourg 1888, frg. P. 5, P. 6, 40 ff. [non vidi]; die dt. Übers. ist abgedruckt in Hild / Hellenkemper (1986) 44-47. Der Heros Sarpedon war der mythische Führer der Lykier im Trojanischen Krieg und wurde später mit Apoll identifiziert; vgl. Otto IMMISCH, s.v. 'Sarpedon' in: Roscher Myth. Lex. 4 (1909-1915) 396-400; Walter RugE, s.v. 'Sarpedon(ion)', $R E$ II A 1 (1921) 48; Johannes ZwICKER, s.v. 'Sarpedon 4', RE II A 1 (1921) 45f.; Magie (1950) II 1142. Nach Hild / Hellenkemper (1986) 44 Anm. 72 wird das Heiligtum des Apollo Sarpedonius sonst nur noch bei Zos. erwähnt. Eine weitere Erwähnung findet sich jedoch bereits bei Diod. XXXII 10,2. Der Diadoche Balas hatte von 150 v. Chr. bis 146 v. Chr. den Thron von Syrien inne. Nach seiner Vertreibung konsultierte er das Orakel nach Chancen für seine Rückkehr an die Macht.

25 Zos. I 57, 2 zitiert darin den negativ ausfallenden Orakelspruch an die Palmyrener; vgl. HartManN (2001) 307f. u. Anm. 178.

26 Zu diesem Komplex vgl. jetzt GotTER (2003). 
Eine weitere spätantike Heiligenvita erzählt von der Christianisierung der Isaurier, besonders der Einwohner von Isaura Nova, durch den Heiligen Konon ,zur Zeit der Apostel". Konon ist neben Thekla der berühmteste isaurische Heilige; er wurde oft zusammen mit ihr abgebildet. ${ }^{27}$ Die Isaurier wurden nach der Vita während einer Kulthandlung für Apollo bekehrt. Der Gott ist auf zwei kaiserzeitlichen Münzen aus lsaura abgebildet; er war noch lange nach dem hier im folgenden geschilderten Bekehrungswunder die Hauptgottheit in Isaura. ${ }^{28}$ Möglicherweise spiegeln sich deshalb in dieser Geschichte auch noch zeitgenössische heidnische Praktiken; allein der Text gibt darauf keinen direkten Hinweis. In der Geschichte begehen bewaffnete Männer das Geburtstagsfest des Apollo und opfern ihm in einer Höhle vier Meilen außerhalb von Isaura Nova, wo sich ein Standbild des Gottes befand ${ }^{29}$ Der Heilige schlägt, um die höhere Macht des christlichen Gottes zu beweisen, einen Wettlauf zum Bildnis vor, den er dank göttlichen Eingreifens gewinnt: Der Himmel verdunkelt sich, und die heidnischen Isaurier verlaufen sich in den zerklüfteten Tälern. Schließlich zwingt Konon sogar die Apollo-Statue selbst dazu, die Arme zu heben und Gott zu preisen. ${ }^{30}$

Verfasser dieser erbaulichen Vita Cononis ist wohl ein Mönch. Dieser wollte zudem nach dem Tod des Heiligen mit einer Geschichte, die von in Amphoren aufbewahrten Dämonen handelte, die isaurischen, nun zumindest in der Mehrzahl christlichen Banditen (wären sie sonst seiner Geschichte zugänglich gewesen?) abschrecken. Die fraglichen Amphoren mit den bereits vom Heiligen Konon eingeschlossenen Dämonen wurden in der Grabeskirche des Heiligen verwahrt. Nach langer Zeit wurde

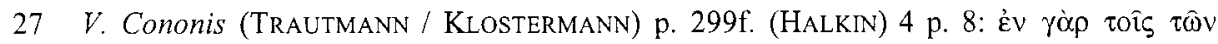

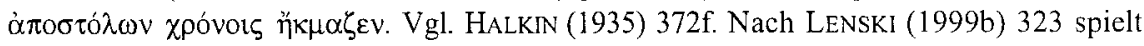
die Vita im frühen 4. Jh. Es sind drei Konon-Legenden überliefert, die sich aus drei verschiedenen gleichnamigen Personen speisten und die bereits im Altertum verwechselt wurden: Konon dem Gärtner, der das Märtyrium im pamphylischen Magydos erlitt, ein isaurischer Konon von Iconium, Märtyrer unter Decius und Aurelian (für beide vgl. Maria-Barbara V. STRITzKY, s.v. 'Konon', LThK3 VI (1997) 274), und der hier genannte Konon von Bidana in Isaurien; vgl. Halkin (1935) 373. (1985) 5, Anm. 2; BUSCHHAusEN (1971) 197 (Abbildungen). Die Passio, BHG 2077, wurde ins Slawische uibersetzt, erhalten im codex Suprasliensis, und in abgekürzter Form bei Michael IV. tradiert (BHG 2078). In Konstantinopel besaß Konon eine Kirche, deren Mönche die Verurteilten des NikaAufstands im Jahr 532 spielten, Mal. p. 474; Theoph. AM 6024, wobei dieser Konon auch mit dem „Gärtner" identifiziert werden kann, vgl. HALkIN l.c. In Syrien wurde eine Reihe von Klöstern höchstwahrscheinlich nach diesem Konon benannt, vgl. GERO (1973) 22, Anm. 47. Auch in Mesopotamien wurde er verehrt; Justinian erneuerte dort ein Armenhaus des Heiligen, Proc. aed. V 9, 35.

$28 C G C 2113 \mathrm{f}$, Nr. 3 u. 4: Apollo mit dem Kaiser.

29 V. Cononis (TRAutmann / KlostermanN) p. 301; das Standbild wertet die andere Fas-

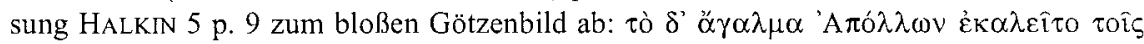
$\dot{\varepsilon} \gamma \chi \omega$ piots.

$30 \quad$ V. Cononis (HaLKIN) 8 p. 12; vgl. SHaW (1990) $246 \mathrm{ff}$. 
eine Amphore in der Hoffnung auf einen darin befindlichen Schatz geöffnet, worauf sich zwei schreckliche Dämonen namens Diomedes und Kouttonios erhoben, die erst wieder durch tagelanges Fasten und Beten der Mönche gebannt werden konnten. ${ }^{31}$ Die Geschichte reflektiert (wie auch die Miracula der Heiligen Thekla) die Bedrohung der Kirchenschätze in Isaurien durch die Banditen. ${ }^{32}$ Aber sie gibt keinen Hinweis auf ein noch zum Zeitpunkt der Abfassung lebendiges und weit verbreitetes Heidentum der Isaurier. Im Gegenteil, wenn die Isaurier zu dieser Zeit noch in der Mehrzahl heidnisch gewesen wären, so hätte dies auch der Intention des Hagiographen widersprochen, der die erfolgreiche Missionierung der Isaurier durch Konon auch im Hinterland bereits zum frühestmöglichen Zeitpunkt postuliert, während der Missionsreise des Paulus.

Gerade aus den immer wieder als Beleg für ein fortgesetztes Heidentum der Isaurier zitierten hagiographischen Quellen geht hervor, daß selbst die schlimmsten isaurischen Räuber sich als Christen sahen, denn sonst wäre ihre Bekehrung zum Christentum in diesen Quellen gewiß explizit erwähnt worden, was freilich niemals der Fall ist. Die folgende Stelle aus der syrischen Vita des älteren Symeon Stylites (390-459), des ersten Säulenheiligen, mag als Beleg hierfür dienen, ${ }^{33}$ auch wenn sie eine ganz andere Absicht verfolgt, nämlich die göttliche Kraft des noch jungen Heiligen unter Beweis zu stellen. Eine isaurische Räubertruppe war während der großen Aufstände nach 400 bis nach Şiş bei Nicopolis in der Euphratesia vorgedrungen, wo der früh erleuchtete Symeon noch seine Jugend verbrachte. Die Räuber führten 400 Bewohner weg, darunter den Heiligen und dessen Neffen. Symeon wurde umgehend durch Gottes Hilfe befreit. Er begab sich aber aufs Neue in das Räuberlager, um die Herausgabe der Gefangenen zu verlangen. Durch ein Wunder entging der Heilige seiner Enthauptung durch die Räuber. Dem einen Isaurierhauptmann mit dem indigenen Namen Bos hauchte er durch Handauflegung einen unreinen Geist ein. Die Räuber waren sich nun nicht sicher, ob er ein Christ sei, und sie forderten ihn auf, ihnen sein Christentum zu bekennen, was auf ihr christliches Bekenntnis schließen läßt. Der Aufforderung kam Symeon umgehend nach und eröffnete ihnen somit seine göttliche Vollmacht über die

31 V. Cononis (Trautmann / Klostermann) p. 320, Z. 6; Trautmann / Klostermann vermuten im Motiv der auf Flaschen gezogenen Dämonen einen orientalischen Einfluß des Autors und bemerken eda. 299 den seltsamen Namen des zweiten Dämonen. Kouttonios erinnert an den indigenen Beinamen des isaurischen heidnischen Banditen Indacus Kottunes und ist vielleicht ein Verweis auf die Urtümlichkeit des Dämons.

32 So deutet HaLKIN (1935) 371 diese ,fables absurdes“.

$33 V$. Simeonis Stylitiae 7, p. 83f. (syrische Vita). Eine weitere Vita ist von seinem Schüler Antonius zu Zenos Regierungszeit verfaßt worden, vgl. KW 293, Nr. 113; Walter BERSCHIN, s.v. 'Antonios 6', DNP 1 (1996) 808. Eine dritte Version bietet Theod. h.r. XXVI. Der Quellenwert der syrischen Version ist höher einzustufen als die Vita des Antonius, vgl. LietzManN $V$. Simeonis (1908) 215-223; auch Euagr. h.e. I 13 lehnt sich an den Syrer an. 
Dämonen. ${ }^{34}$ Als dies geschehen war, bat ihn Bos sogleich, daß der Heilige für ihn beten solle - ein hagiographischer Topos, wie die oben zitierte Theodoret-Stelle zeigt, wo ebenfalls isaurische Banditen den Heiligen bitten, daß er für sie bete. ${ }^{35}$ Doch wiederum bleibt ein Heidentum der Isaurier unerwähnt, von ihrem Christentum wird also stillschweigend ausgegangen. Der andere isaurische Anführer hieß Altemdorus, wohl eine Korruption für Artemidorus. Auffallend ist hierbei die Parallele zu den beiden Dämonen Diomedes und Kouttonios aus den Miracula, wo ebenfalls ein indigener Name neben einem griechischen steht.

Wenn ein Isaurier dennoch heidnischen Glaubens war, so wird dies gerade in den hagiographischen Quellen ausdrücklich als Ausnahme geschildert. Bei einem in der Vita des jüngeren Symeon Stylites geschilderten Wunder von der Heilung eines Gelähmten wird dieser von seinen isaurischen Landsleuten unmittelbar nach seiner Heilung in deren (christliches!) Oratorium gebracht. ${ }^{36}$

Auf der anderen Seite wurde in der Forschung zuweilen versucht, das isaurische Heidentum soziologisch von den Isaurieraufständen her zu erklären. Demnach habe sich vor allem in den unteren Volksmassen, die vermehrt der Räuberei zugeneigt hätten, heidnischer Glauben lange gehalten. ${ }^{37}$ Doch diese Hypothese läßt sich ebenso aus den Quellen heraus widerlegen, denn auch sozial hochrangige Isaurier waren noch im 5. Jahrhundert Heiden. Neben dem oben erwähnten Banditen Indacus, gab es jenen dem Kaiser gegenüber loyalen isaurischen Krieger, den ersten Zeno, sowie eine Reihe von isaurischen Gelehrten wie Marsus. ${ }^{38}$ Ein Zeitgenosse dieses Zeno, der hier schon oft genannte Theodoret, schreibt ihm als Bischof von Kyrrhos, er möge ,nach der Bekleidung des Konsulats auch das heilige und göttliche Kleid [also das Taufkleid] annehmen", und auf einem Fußbodenmosaik in Seleucia läßt sich der Heermeister als „Ares-Freund“ darstellen, was ein Hinweis auf seinen beruflichen und religiösen Standpunkt ist, und Damascius nimmt ihn in seinen Heiden-Katalog auf. ${ }^{39}$ Zudem werden in den Miracula auch isaurische Prominente erwähnt, welche die Heilige als

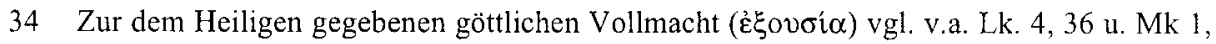
22. 2, 10. 3, 15. 6, 7. 13, 34; Mt. 7, 29. 9, 29. 10, 1; Lk 5, 24. 9, 1. 10, 11; 2 Kor. 13, 10.

35 Theod. h.r. X 5. Anders Trombley (1994) II 127: Symeon hätte nicht von sich gesagt, daß er Christ sei, wenn die Isaurier keine Heiden gewesen wären.

36 V. Simeonis Stylitiae Iunioris 188f. mit VAN DEN VEN V. Simeonis II (1970) 192, Anm. 4.

37 LENSKI (1999b) 324f. argumentiert in diese Richtung, ebenso CHAUvot Priscian, Paneg. (1986) 122 und in dessen Gefolge HEUCKE (1997) 53.

38 Hierzu s.u. Kap. V.1.3.

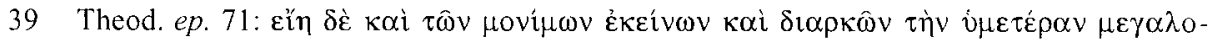

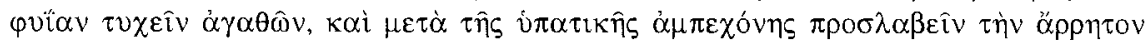

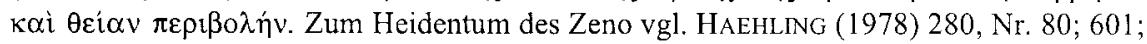

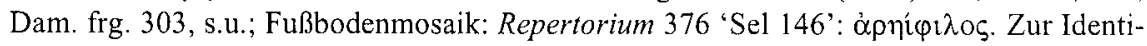
fikation des Fußboden-Epigramms mit diesem Zeno vgl. ŞAHIN (1991) 159. Näheres u. voller Text s.u. Kap. V.1.2. 
Christen verehrten. Ein Beispiel gibt Bassiane, eine isaurische Adlige, die nach den kriegsähnlichen Auseinandersetzungen zwischen Isauriern und Römern in der Mitte des 4. Jahrhunderts vergeiselt und im Thekla-Heiligtum bei Seleucia festgehalten wurde. Die Schwangere vertrug das feuchtheiße Klima an der Küste nicht und fiel in Ohnmacht, wurde jedoch von der Heiligen gerettet. Ihr Sohn Modestus soll es im isaurischen Eirenopolis zu einem angesehenen Bürger gebracht und die Wundergeschichte dort publik gemacht haben. ${ }^{40}$

Andere prominente Isaurier waren zwar christlichen Glaubens, zeigten jedoch, wie viele spätantike Gelehrte, offene Sympathien für den Neoplatonismus. Hier ist Illus mit seinen unverhohlenen Sympathien für den heidnischen Philosophen Pamprepius zu nennen, der sein Günstling wurde. ${ }^{41}$ Bei Illus liegt dabei, ebenso wie bei dem ersten Zeno, eher das Bestreben zugrunde, dem durch die räuberischen Aktivitäten der vorausgegangenen Jahrzehnte erworbenen Ruf als Barbar zu entkommen. Dies sollte durch den Kontakt zur griechisch-römischen Gelehrsamkeit bewerkstelligt werden. ${ }^{42}$ Insofern ist die Interpretation einer vom zeitgenössischen, neuplatonischen Autor Damascius genannten Liste heidnischer Empörer, der erste Zeno sei in religiöser Hinsicht ein Vorläufer des Illus, des isaurischen Rivalen des Kaisers Zeno, irreführend. ${ }^{43}$ Diese Aufzählung beginnt mit Kaiser Julian Apostata und endet mit Illus. Man nahm die Liste sogar zum Anlaß, eine tatsächlich erfolgte heidnische Revolte zu dieser Zeit anzunehmen. Dies ist jedoch schon aufgrund der Tatsache abwegig, daß bei der von dem Christen Illus betriebenen Krönung des Usurpators Leontius im Jahr 484 dessen Rechtgläubigkeit eigens herausgestellt wurde, auch wenn es sich um eine übliche Formel bei Krönungszeremonien gehandelt haben mag. ${ }^{44}$

40 Mir. 19 mit Dagron (1978) $120 \mathrm{f}$.

41 Zu Pamprepius vgl. Asmus (1913), der freilich das von Delatte / Stroobant (1923) publizierte ,Rhetorius“-Horoskop noch nicht kannte, vgl. PINGREE (1976) 144-147; Rudolf KeYdell, s.v. 'Pamprepios', RE XVIII 2 (1949) 409-415; PLRE II 825-828 s.n. 'Pamprepius'; Kaster (1988) 329-332, Nr. 114; Athanassiadi (1993); Salamon (1996) (polnisch mit englischem Abstract); FELD (2002).

42 S.u. Kap. V.1.3.

43 Dam. frg. 303; Haehling (1980) 92-95 gegen ThOMPSON (1946) 28f.; s.u. - Dam. verfaßte seine Vita Isidori zwischen 493 und 526: Dam. frg. 94; PLRE II 342f. s.n. 'Damascius 1'; ZINTZEN Damascius (1967) IX. Zu seiner Bedeutung als Philosoph: Asmus (1913) passim; Reinhold StrömBERG, 'Damascius. His personality and significance' in: Eranos 44 (1946), 175-192; ATHANASSIAdi (1993) passim; Marco S. ToRINI, 'Damaskios oder Vom heulenden Nordwind der Notwendigkeit' in: Gerald Hartung / Wolf Peter KLEIN (Hrsg.), Zwischen Narretei und Wahrheit. Biographische Skizzen und Konturen alter Gelehrsamkeit, Hildesheim-Zürich-New York 1997, 61-94.

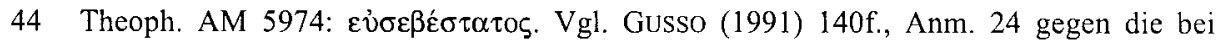
JARRY (1968) 153 formulierte These einer tatsächlich stattgefundenen ,insurrection pagano-chalcédonienne"; ebenso CHUVIN $\left({ }^{2} 1991\right)$ 100, 102: „De fait, la révolte d'Illous s'inscrit dans les manœvres des Isauriens pour s'assurer le pouvoir suprême et ses aspects 
Bemerkenswert ist immerhin die hohe Zahl an Isauriern in dieser Liste: neben dem ersten Zeno sind Severianus, der vermutlich ebenfalls Isaurier war, sowie Marsus und Illus genannt. ${ }^{45}$ Doch erweckt die Aufzählung einigen Argwohn angesichts ihres konstruierten Aufbaus, zweitrangige Gelehrte und Beamte und deren auf einen kleinen Wirkungskreis begrenzten Widerstand in einem Atemzug mit dem großen Kaiser Julian zu nennen. So ist sie eher als ein absichtsvoller Versuch denn als eine glaubwürdige Beschreibung einer historischen Gegebenheit zu interpretieren. Entweder versuchte Damascius, mit Blick auf die christliche Oberschicht, nach den Ereignissen in Ägypten (dort hatte Pamprepius im Auftrag des aufrührerischen Illus versucht, die heidnischen Philosophen für dessen Sache einzuspannen ${ }^{46}$ den nun wohl bestehenden Illoyalitätsvorwurf an die Heiden dadurch zu entkräften, daß er die heidnischen Usurpations- und Restaurationsversuche als folgenlos und zweitrangig für die Geschicke des Imperiums darstellte. ${ }^{47}$ Oder er wollte für seine heidnischen Leser eine kleine Geschichte des heidnischen Widerstands konstruieren, und vor dieser Folie Pamprepius wegen seines Scheiterns in Ägypten und der destruktiven Wirkung auf die Philosophen als einen hochgefährlichen Verräter an dem Fernziel einer heidnischen Renaissance brandmarken. ${ }^{48}$

Den in den Quellen vereinzelten und sich auf singuläre Ereignisse beziehenden Nachrichten über heidnische Individuen oder heidnische Feste steht die Existenz einer übergroßen Zahl von isaurischen Bistümern entgegen, was auf eine vorrangig christliche Bevölkerung in der Region hindeutet. Jede noch so kleine Ortschaft der isaurischen Dekapolis im Kalykadnostal hatte einen Bischof; die Teilnehmerliste des Konzils von Seleucia und die Endnotiz einer Bistumsliste führen für die Isauria nicht weniger als 24 Suffraganbistümer an, deren Zahl sich für die Zeit zwischen dem

religieux restent secondaires [...] L'ambition domine toute cette histoire et non la religion"; diese Interpretation auch schon bei Johannes GefFCKEN, Der Ausgang des griechisch-römischen Heidentums, Heidelberg 21929, ND Darmstadt 1963, 196 und Anm. 209; insgesamt hierzu SALAMON (1996) 192-195 gegen TROMBLEY I (1993) 81 ff. HaEHLING (1980) 94 modifiziert dies dahingehend, daß die Religiosität der Bevölkerung immer als Gradmesser für die Loyalität zum Staat verstanden worden sei. Auch STEIN (1949) 23 sieht die Orthodoxie und die Reste des Heidentums vereint im Kampf gegen den Monophysitismus.

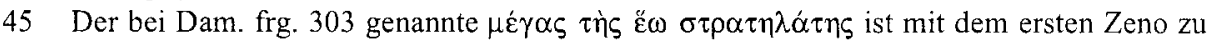
identifizieren, vgl. THOMPSON (1946) 28f.; DemandT (1970) 743; PLRE II 1200 s.n. 'Fl. Zenon 6'; s.u. V.1.2.

46 S.u. Kap. V.2.3.

47 Dam. frg. $303=$ Epit. Phot. 290. Zu dieser Deutung der Intention des Damascius gelangt HAEHLING (1980) 92-95. Allerdings schenkt er der Tatsache, daß Damascius eher für eine heidnische als eine christliche Zielgruppe geschrieben hat, keine Beachtung.

48 So die ältere Deutung von Asmus (1913) $341 \mathrm{ff}$. 
Chalkedonense 451 und dem Ende der Autokephalie 787 auf 41 erhöhte. ${ }^{49}$ Die in der Spätantike entstandenen Wundergeschichten über die isaurischen Lokalheiligen Thekla und Konon reflektieren zwar die Bedrohung der Kirchenschätze durch die isaurischen Banditen. Doch daß diese Raubzüge durch deren Heidentum motiviert gewesen sein sollen, wird in den Hagiographien nirgends behauptet oder auch nur insinuiert. Vielmehr dürfte die Intention der Autoren anders gelagert gewesen sein: ebenso wie die Heiligen zu ihren Lebzeiten gegen das Heidentum gewirkt haben, so wird ihre segensreiche und schützende Wirkung gegen die Räuber der Gegenwart im 4. und 5. Jahrhundert dargestellt und damit erhofft. Über deren religiöse Orientierung in der Spätantike jedoch geben die Hagiographen kaum Auskunft, und für die Feststellung von Noel LENSKI, die isaurischen Banditen aus dem Hochland des 4. Jahrhunderts seien auch durch aggressives Heidentum motiviert gewesen, ${ }^{50}$ gibt es, wie wir gesehen haben, keine ausreichende Quellengrundlage. Somit ließe sich LENSKIs Behauptung umkehren: Viele Isaurier waren bereits früh christianisiert, einige blieben jedoch bis in die Spätantike heidnisch. Nach Sicht der Hagiographen stellte sich ohnehin jeder, der sich gegen die christlichen Kultstätten richtete, außerhalb der christlichen Glaubensgemeinschaft.

Die Frage, ob die isaurischen Banditen nun Christen oder Heiden waren, wird damit zweitrangig. Die Religion läßt sich nicht als bestimmendes Unterscheidungskriterium oder als Erklärung für den fortgesetzten isaurischen Widerstand heranziehen. ${ }^{51}$ Heidnische Praktiken in allen gesellschaftlichen Schichten, sowohl bei der niederen Bevölkerung als auch bei hohen Beamten, die von der Obrigkeit toleriert werden, finden sich im gesamten Reich noch bis in das späte 5. Jahrhundert, so im karischen Aphrodisias. ${ }^{52}$ Auch Pamprepius bekleidete 479 das Amt des quaestor sacri

49 Notitia 3, 85 (Autokephalie). 508-533 (Isaurien), 4, 493 (Endnotiz) mit DarrouzĖs (1981) 29. Die Bischofslisten bei RAmSAY (1890) [Faltblatt]; Jones $\left({ }^{2} 1971\right)$ 541, Appendix 4; Bei DarrouzĖs Notitiae (1981) 30 eine Tabelle der isaurischen Bistümer und Abgleich mit Hierocl. und Georg. Cypr.

50 LENSKI (1999b) 323 ff. versucht, die u.a. bei Basilius bezeugte Existenz isaurischer Bistümer mit den Nachrichten über Heidentum und Räuberei mit den Worten in Einklang zu bringen: „As elsewhere in the empire, some of the population was Christian; many others remained pagan." Alle Hochlandbewohner jedoch hätten eine Trennlinie zwischen sich und den Bewohnern der Ebene gezogen, ob sie nur heidnisch blieben, sich äußerer Kontrolle ihres Bistums entzogen oder ,by actively forcing their pagan beliefs on those around them."

51 Zur weiteren Diskussion um die Gründe des sich in fortgesetzten Raubzügen äußernden isaurischen Widerstands gegen Konstantinopel s.u. Kap. IV.3.

52 Dam. frg. 185 = Suda A 4174; Zach. Rhet. $V$. Severi pp. 14. 16 ff. 22.39 ff.; vgl. PLRE II 160 ff. s.n. 'Asclepiodotus 3'. 831 s.n. 'Paralius'; zu den Ausgrabungen Charlotte RoueCHÉ, Aphrodisias in Late Antiquity: The late Roman and Byzantine inscriptions including texts from the excavations at Aphrodisias, London 1989. 
palatii unter dem isaurischen Kaiser Zeno. ${ }^{53}$ Der quaestor Isocasius, ein gelehrter Heide aus Antiochia, wurde unter Leo I. des Heidentums bezichtigt, doch er bekam noch die Chance einer Anhörung vor dem Senat; zuvor hatte er das Thekla-Heiligtum besucht. ${ }^{54}$ Heiden in hohen Ämtern wurden erst unter Justinian verboten, speziell im Quaestorenamt haben sie sich lange gehalten. ${ }^{55}$ Die Anordnung des Theodosius aus dem Jahr 380, daß im Zuge der Erhebung des Christentums zur Staatsreligion alle Heiden aus dem Heeresdienst zu entlassen seien, war offenbar wirkungslos geblieben. $^{56}$

53 S.u. Kap. V.2.3.

54 Mal. p. 369, 371; Theoph. AM 5960; PLRE II 633f. s.n. 'Isocasius'; KASTER (1988) 301f., Nr. 85; Mir. 39 mit Dagron (1978) $91 \mathrm{f}$.

55 CJ I 11, 9f.; Karl Leo Noethlichs, s.v. 'Hofbeamter', RAC XV (1991) 1111-1158. 1139f.; Harl (1990) 22f.; Elton (2000b) $403 \mathrm{f}$.

56 CTh XVI 1, 2. 10, 21; vgl. Demandt (1970) 763. 